\title{
Impact of Systemic Lupus Erythematosus on Ovarian Reserve in Premenopausal Women before Receiving Cyclophosphamide Therapy: Evaluation Using Anti-Müllerian Hormone
}

\author{
Weixia Wei1,2, Qi Lin³, Qianyu Huang1,2, Huiru Tang1,2, Liping Wang1,2, Guoping Wang1,2, \\ Junxia Zhou ${ }^{1,2}$, Ruifang Wu ${ }^{1,2}$, Qingwen Wang ${ }^{3}$, Ruiying Diao ${ }^{*}$ \\ ${ }^{1}$ Department of Obstetrics and Gynecology, Peking University Shenzhen Hospital, Shenzhen, China \\ ${ }^{2}$ Shenzhen Technical Research and Development Center on Gynecologic Oncology, Peking University Shenzhen \\ Hospital, Shenzhen, China \\ ${ }^{3}$ Department of Rheumatology and Immunology, Peking University Shenzhen Hospital, Shenzhen, China \\ ${ }^{4}$ Reproductive Medicine Centre, Shenzhen Secondary Hospital, First Affiliated Hospital of Shenzhen University, \\ Shenzhen, China \\ Email: *15889753127@163.com
}

Received 10 January 2016; accepted 22 February 2016; published 25 February 2016

Copyright (C) 2016 by authors and Scientific Research Publishing Inc.

This work is licensed under the Creative Commons Attribution International License (CC BY). http://creativecommons.org/licenses/by/4.0/

(c) $\underset{\mathrm{EY}}{\mathrm{i}}$ Open Access

\section{Abstract}

Introduction: Anti-Müllerian hormone (AMH) is shown to be a possible indicator of ovarian function. Severe systemic lupus erythematosus (SLE) patients exposed to high-dose cyclophosphamide (CTX) have a much higher risk of developing infertility and premature ovarian failure. Therefore, we performed a prospective case-control study to evaluate the impact of SLE on women's ovarian reserve using AMH before CTX therapy. Methods: SLE patients before receiving CTX therapy were enrolled in our hospital. Age-matched healthy women were served as controls. Serum AMH level was measured using an enzyme-linked immunosorbent assay. Basal hormone levels were measured including follicle-stimulating hormone, luteinizing hormone, and estradiol on the third day of their menstrual periods. All participants underwent transvaginal ultrasonographic examination for the determination of total antral follicle count on the third day. Results: AMH value in SLE patients was significantly lower compared to healthy control with normal ovarian reserve. No significant difference in AMH levels was found between SLE and healthy control with low ovarian reserve. Conclusions: SLE patients not receiving CTX therapy even with normal menstruation, still

*Corresponding author.

How to cite this paper: Wei, W.X., et al. (2016) Impact of Systemic Lupus Erythematosus on Ovarian Reserve in Premenopausal Women before Receiving Cyclophosphamide Therapy: Evaluation Using Anti-Müllerian Hormone. Advances in Reproductive Sciences, 4, 17-22. http://dx.doi.org/10.4236/arsci.2016.41003 
had an impaired ovarian reserve. Therefore, early monitoring of AMH levels could better reflect the ovarian function and reproductive outcomes of SLE patients and relative protective strategy needed to reserve fertility.

\section{Keywords}

\section{Anti-Müllerian Hormone (AMH), Ovarian Reserve, Cyclophosphamide (CTX), Systemic Lupus Ery- thematosus (SLE)}

\section{Introduction}

Ovarian reserve is a key determinant of fertility preservation and endocrine function of reproductive-aged women. At present, potential predictions of ovarian reserve include age, Follicle-stimulating hormone (FSH) at early follicular phase (Day 2 - 5), Luteinizing hormone (LH), Estradiol (E2), Inhibin B, Antral follicle count (AFC) and AMH. Among them, serum AMH can help evaluate the progression of ovarian senescence, as it is independent of hypothalamic-pituitary-gonadal axis role and will decrease to undetectable levels at menopause

[1]. For example, serum AMH levels correlate with the extent of gonadal damage in cancer survivors [1].

Systemic lupus erythematosus (SLE) is a chronic autoimmune systemic disease that mainly affects women of reproductive age (20 to 40). Theoretically, pulsed intravenous cyclophosphamide (CTX) is considered as standard therapy for severe SLE. Unfortunately, emerging data have shown that ovarian reserve and fertility are diminished even in the mild disease, indicating a direct impact of SLE itself on ovarian function [2]. Further severe impact in SLE patients is the therapy strategy using alkylating chemotherapy agent CTX. For example, the frequency of SLE patients with Systemic Lupus Erythematosus Disease Activity Index (SLEDAI) $\geq 8$ is found to be significantly higher in patients with irregular cycles than those with regular cycles, indicating that the high SLEDAI levels are associated with impaired ovarian function [3].

While the therapy rate of CTX on SLE is substantial, many concerns are about its side effects or toxicity. In addition to the immuno-suppression, bone marrow suppression, alopecia, hemorrhagic cystitis and malignancy, ovarian failure is an important side effect associated with the use of CTX. Other research shows that, the subclinical impairment of ovarian reserve can be found in the SLE patients, even with normal menstruation and short illness duration [4]. Specifically, the risk of sustained amenorrhea or ovarian failure depends on the age of SLE patients and the cumulative dose of CTX [5]. Therefore, this study aims to monitor ovarian function of SLE patients comprehensively by monitoring the AMH levels before CTX treatment.

\section{Materials and Methods}

\subsection{Subjects}

This study was approved by the Institutional Review Board of Peking University Shenzhen Hospital, and informed written consent was obtained from all the individuals. Twenty-seven severe SLE female patients were not receiving CTX treatment before and enrolled in our hospital between Match 2013 and December 2014 (as Group A). At the same time, 50 healthy patients were also recruited as control, with a mean age that matched with the Group A. The control was further divided into two groups: Group B with low ovarian function $(\mathrm{n}=18)$ and Group C with complete ovarian function $(\mathrm{n}=32)$. Classification criteria of Group B and C was based on concomitant assessments by measuring hormone serum levels, including $\mathrm{FSH}, \mathrm{LH}$ and $\mathrm{E}_{2}$ during the follicular phase (second to fifth day) of menstrual cycle and evaluating AFC (approximate diameter: 2 - $9 \mathrm{~mm}$ ) by vaginal ultrasound. Specifically, Group B with decline in ovarian reserve (DOR) was defined as FSH>10IU/L and bilateral $\mathrm{AFC}<6$.

All the patients and control volunteers were $\leq 40$ year old, and had a normal menstrual cycle (defined as flow duration of 3 - 7 days and cycle duration of 21 - 35 days). Treatment with the following drugs was allowed in patients of Group A, including glucocorticosteroids, mycophenolate mofetil, hydroxychloroquine and methotrexate. None of the patients was diagnosed with polycystic ovarian syndrome, or had a history of ovarian surgery or previous cytotoxic drug therapy. Age of onset, duration of illness, SLEDAI, current medications and previous treatment were recorded. 


\subsection{Serum AMH Assays}

The AMH serum level was measured using a repeated enzyme-linked immunosorbent assay (Human antiMüllerian hormone ELISA Kit, CSB-E12756h, Biotechnologies, Inc., CHN). AMH normal range was in 1 - 8 $\mathrm{ng} / \mathrm{mL}$, while value $<1 \mathrm{ng} / \mathrm{mL}$ was regarded as reduced. All the serum samples were tested at the same time to minimize day-to-day assay variation. Intra- and inter-assay coefficients of variation were limited to $3.0 \%$ and $6.0 \%$, respectively.

\subsection{Statistical Analysis}

Statistical analyses were performed using the social sciences (SPSS) software package (ver. 13.0; SPSS Inc.). Values were expressed in mean \pm S.E.M. Differences in data between groups were determined using one-way analysis of variance. For all the analyses, $\mathrm{P}<0.05$ was defined as statistically significant.

\section{Results}

As shown in Table 1, the average age and mean duration of illness in the SLE patients were 28 years (27.9 \pm 5.50) and 15 months $(15.0 \pm 9.70)$, respectively. The mean SLEDAI in the SLE patients was $12.1 \pm 1.6$ (Table 1). Clinical characteristics of 27 SLE patients included renal disease, pulmonary hypertension, arthritis and vasculitis, and CTX treatment would be needed. They had the history of corticosteroids prednisone $(1 \mathrm{mg} / \mathrm{kg} / \mathrm{d})$ treatment. And 23 cases had been treated with other immunosuppressive drugs (single or combined) (Table 1).

The average age was similar between the study and control groups $(\mathrm{P}=0.053$, Table 2$)$. The decline in ovarian reserve (DOR) is defined as FSH $>10 \mathrm{IU} / \mathrm{L}$ and bilateral $\mathrm{AFC}<6$. The basal average levels of serum FSH were comparable between SLE patients (Group A, $13.05 \pm 1.94$ ) and Group B (13.94 \pm 2.27$)$, but both were significantly higher than normal control with complete ovarian function (Group C, $6.54 \pm 1.57, \mathrm{P}<0.01$, Table 2). The value of total AFC was similar between SLE patients (Group A, $4.48 \pm 1.48$ ) and Group B (4.39 \pm 1.68 ), and both were significantly lower than Group C (11.03 \pm 2.12 , P $<0.01$, Table 2). As shown in Table 2, the levels of serum $\mathrm{LH}$ and $\mathrm{E}_{2}$ were comparable between the three groups $(\mathrm{P}>0.05)$.

Notably, as shown in Figure 1, the AMH level in SLE patients (Group A, $2.07 \pm 0.34$ ) was slightly higher than Group B (1.22 $\pm 0.14, \mathrm{P}>0.05)$, but was significantly lower than Group C $(4.79 \pm 0.42, \mathrm{P}<0.001)$. It was indicated that the ovarian reserve function may be lower in the patients with severe SLE patients, even with normal menstruation. And the SLE patients had comparable ovarian reserve function with DOR. Therefore, early monitoring of AMH could better reflect the ovarian reserve and reproductive outcomes of female patients with SLE.

Table 1. Clinical characteristics of 27 SLE patients.

\begin{tabular}{lc}
\hline & Values \\
\hline Mean age (years) & $27.9 \pm 5.5$ \\
Mean duration of disease (years) & $15.0 \pm 9.7$ \\
Mean SLEDAI & $12.1 \pm 1.6$ \\
SLE clinical features & $17(62.9 \%)$ \\
Nephropathy & $5(18.5 \%)$ \\
Pulmonary hypertension & $13(48.1 \%)$ \\
Arthritis & $11(0.7 \%)$ \\
Vasculitis & $27(100 \%)$ \\
Corticosteroid therapy (current use) & $23(85.1 \%)$ \\
Other previously used immunosuppressive drugs & $12(44.4 \%)$ \\
Anti-malaria therapy & $5(18.5 \%)$ \\
Mycophenolate mofetil & $0(0 \%)$ \\
Methotrexate & $0(0 \%)$ \\
Azathioprine & \\
Cyclophosphamide (CTX) &
\end{tabular}




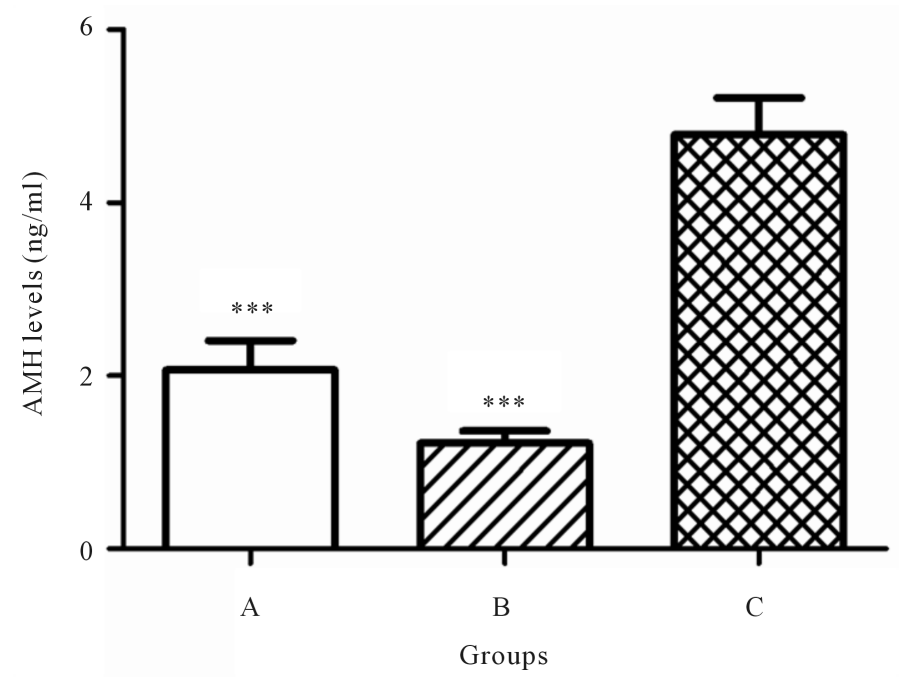

Figure 1. Comparison of AMH levels in three groups. Note: A, SLE patients; B, Control with low ovarian reserve; C, Control with complete ovarian reserve. ${ }^{* * *} \mathrm{P}<0.001$, compared with Group C.

Table 2. Comparison of ovarian reserve function in three groups.

\begin{tabular}{ccccccc}
\hline Groups & $\mathrm{n}$ & Age & FSH (IU/L) & LH (IU/L) & $\mathrm{E}_{2}(\mathrm{pg} / \mathrm{ml})$ & AFC (bilateral) \\
A & 27 & $27.9 \pm 5.5$ & $13.05 \pm 1.94^{* *}$ & $6.30 \pm 2.27$ & $42.48 \pm 7.96$ & $4.48 \pm 1.48^{* *}$ \\
B & 18 & $30.1 \pm 4.4$ & $13.94 \pm 2.27^{* *}$ & $6.22 \pm 2.72$ & $41.41 \pm 9.85$ & $4.39 \pm 1.68^{* *}$ \\
C & 32 & $26.8 \pm 3.5$ & $6.54 \pm 1.57$ & $6.32 \pm 2.57$ & $41.66 \pm 11.11$ & $11.03 \pm 2.12$ \\
\hline
\end{tabular}

Note: A, SLE patients; B, Control with low ovarian reserve; C, Control with complete ovarian reserve. ${ }^{* * *} \mathrm{P}<0.01$, compared with Group C.

\section{Discussion}

With the increase of long-term survival rate of SLE patients, the problem of how to protect the fertility of female patients and improve the quality of life is increasingly concerned [6]. Severely ovarian function and other reproductive failures in SLE patients caused by subsequent CTX therapy would need more protective strategies to preserve their fertility [5] [7]. However, no effective real-time monitoring methods are available to evaluate their fertility and age of menopause. Furthermore, no accepted definition of decreased ovarian reserve is formulated now. Therefore, a better, sensitive and specific method would be needed to monitor the ovarian reserve function in the early stage of SLE treatment, so as the clinician can initiate SLE patients' ovarian protection as early as possible. Previous observations indicated that fertility-preservation options can be offered to some patients with cancer and those at risk of early menopause, such as those with familial cases of primary ovarian insufficiency.

In most cases, premature ovarian failure (POF) exists in SLE female patients because of autoimmune ovarian inflammation or damage caused by specific antibodies due to SLE [8]. Specifically, some unknown mechanism leads to steroid autoimmune lymphocytic ovarian inflammation in SLE patients, resulting in the infiltration of autoimmune antibody into ovarian follicular membrane, even with normal ultrasound appearance in the sinus follicle. Moreover, ovarian dysfunction such as a primary ovarian insufficiency can also be found in SLE patients, as well as multiple glandular syndrome, myasthenia gravis or rheumatoid arthritis [9] [10]. The disorder usually leads to sterility, and has a large effect on reproductive health. Available evidence concerning the performance of ovarian reserve tests is limited by small sample sizes, heterogeneity among study design, analyses and outcomes, and the lack of validated outcome measures. Therefore, it is not wise to initiate the fertility protection just using menstrual cycle changes, because they often occur in the late stage of premature ovarian failure. Currently, the ovarian reserve function in SLE patients is evaluated using basal FSH, LH, $\mathrm{E}_{2}$ and AFC [11]. Among them, the ultrasound AFC was the most reliable test to show the menstrual irregularity and negatively correlated with age. Furthermore, it can more accurately reflect the ovarian reserve function of patients 
with SLE than FSH, $\mathrm{E}_{2}$, LH and ovarian volume. Therefore, ovarian reserve function of the SLE patient can be monitored preliminarily by AFC [12].

Administration of chemotherapy to premenopausal women shortens their reproductive lifespan by depleting non-renewable oocytes. Cytotoxic treatment may accelerate depletion of the primordial follicle pool, leading to impaired fertility and premature menopause. Preservation of fertility is a priority for such women, so identification of women at risk of infertility is important. However, age is the only patient characteristic currently recognized to be predictive of long-term ovarian function after chemotherapy. Pretreatment serum AMH, FSH, antral follicle count, and age predicted late ovarian activity by univariate analysis. Instead, only AMH was predictive in a multivariate logistic regression. Measurement of AMH at cancer diagnosis predicts long-term ovarian function after chemotherapy. Use of this in clinical practice may allow better prediction of chemotherapy-related risk to future fertility. Data from the malignant tumor patients after chemotherapy, pretreatment serum AMH can predict the ovarian reserve function compared to FSH or AFC [13] [14]. AMH is therefore a clinically useful marker of damage to the ovarian reserve in girls receiving treatment for cancer. Because the AMH is secreted by the non-selected follicles (Grade two, sinus and early sinus) with $<6 \mathrm{~mm}$ of the diameter, reflecting directly the size of follicle pool [1]. Moreover, since AMH reflects the number of follicles in the sinus and is not affected by the gonadotropin, the relative stability between the follicular cycle and the cycle is not changed. Therefore, it is a more reliable prediction of ovarian reserve. In this study, the pretreatment FSH level in severe SLE patients was significantly higher than that in healthy control with normal ovarian reserve, but similar to that in control with low ovarian reserve. Nevertheless, the pretreatment AFC and AMH were significantly lower than those in control with normal ovarian reserve, while slightly higher than those in control with low ovarian reserve. FSH, AMH and AFC were detected in the early follicular phase of the SLE female patients with normal menstrual regularity. Although no clinical menstrual changes, the ovarian reserve function had been subclinically damaged, with less AFC and more AMH variation range [15]. Few study focuses on the analysis of pretreatment ovarian reserve function of SLE before CTX treatment. Considering the firts-use of CTX in the non menstrual period of SLE patients, no conventional methods will be avaliable to assess the ovarian reserve function. Moreover, the SLE patients without previous CTX treatment were usually serious and highly active, so it was urgent to immplement the pulsed CTX treament as soon as possible. This is interesting because AMH detection would be suitable for evaluating the ovarian reserve function of SLE patients with different menstrual cycle, regardless of the menstrual period.

In the present study, the ovarian reserve function of severe SLE patients (SLEDAI value was 12.1) was significantly lower than that in normal control, indicating that SLE disease itself had a negative effect on ovarian function. No correlation was found between AMH value and the duration of illness or SLEDAI as an indicator of SLE disease activity in SLE patients without CTX therapy [16]. However, it could be a sign that SLE itself has a negative influence on the ovarian reserve [16]. Our work has shown that the AMH can also be applied as a predictor risk of POF during CTX therapy, especially for female SLE patients with low AMH when first using CTX or for those with fertility requirements. Therefore, regular monitoring of ovarian function would be needed when using CTX treament. Besides, other alternative drugs with low inhibition on gonad would be used as soon as possible during the disease remission. And the patients would better have a baby during this period, or else CTX drugs would lead to further damage on ovarian funciton as the disease progress. Due to the limited sample size, the SLE patients with normal menstruation were not divided further according to the age. Next study needs to focus on the age of SLE patients, so as we can guide the follow-up individualized treatment.

\section{Conclusion}

Together, these data suggest that AMH levels can be used as a sensitive marker for the ovarian reserve function of SLE patients before immunosuppressive treatment. It will help guide rheumatology clinicians to select immunosuppressive drugs and help patients to develop ovarian conservation strategies. For example, immunosuppressive drugs is used when combining with gonadotropin-releasing hormone analogue (Gonadotropin Releasing Hormone Analogs, GnRHa) at the same time or eggs or embryos frozen storage, especially when we have to use CTX.

\section{Acknowledgements}

This work was supported by grants from the National Natural Science Foundation of China (grant number 
81401258), Guangdong Natural Science Foundation (grant number 2014A030313652), Shenzhen Foundation of Science and Technology (grant number JCYJ20150330102401083 and JCYJ2013040211431705), Medical Scientific Research Foundation of Guangdong (grant number A2015122) and Clinical Doctor-Basic Scientist Combination Foundation of Shenzhen Second People's Hospital.

\section{References}

[1] Visser, J.A., Schipper, I., Laven, J.S. and Themmen, A.P. (2012) Anti-Mullerian Hormone: An Ovarian Reserve Marker in Primary Ovarian Insufficiency. Nature reviews Endocrinology, 8, 331-341. http://dx.doi.org/10.1038/nrendo.2011.224

[2] Pasoto, S.G., Mendonca, B.B. and Bonfa, E. (2002) Menstrual Disturbances in Patients with Systemic Lupus Erythematosus without Alkylating Therapy: Clinical, Hormonal and Therapeutic Associations. Lupus, 11, 175-180. http://dx.doi.org/10.1191/0961203302lu163oa

[3] Fatnoon, N.N., Azarisman, S.M. and Zainal, D. (2008) Prevalence and Risk Factors for Menstrual Disorders among Systemic Lupus Erythematosus Patients. Singapore Medical Journal, 49, 413-418.

[4] Ma, W., Zhan, Z., Liang, X., Chen, J., Huang, X. and Liao, C. (2013) Subclinical Impairment of Ovarian Reserve in Systemic Lupus Erythematosus Patients with Normal Menstruation Not Using Alkylating Therapy. Journal of Women's Health, 22, 1023-1027. http://dx.doi.org/10.1089/jwh.2013.4255

[5] Katsifis, G.E. and Tzioufas, A.G. (2004) Ovarian Failure in Systemic Lupus Erythematosus Patients Treated with Pulsed Intravenous Cyclophosphamide. Lupus, 13, 673-678. http://dx.doi.org/10.1191/0961203304lu2012oa

[6] Skomsvoll, J.F., Ostensen, M., Baste, V. and Irgens, LM. (2001) Number of Births, Interpregnancy Interval, and Subsequent Pregnancy Rate after a Diagnosis of Inflammatory Rheumatic Disease in Norwegian Women. The Journal of Rheumatology, 28, 2310-2314.

[7] Oktem, O., Guzel, Y., Aksoy, S., Aydin, E. and Urman, B. (2015) Ovarian Function and Reproductive Outcomes of Female Patients with Systemic Lupus Erythematosus and the Strategies to Preserve Their Fertility. Obstetrical \& Gynecological Survey, 70, 196-210. http://dx.doi.org/10.1097/OGX.0000000000000160

[8] Wheatcroft, N.J., Salt, C., Milford-Ward, A., Cooke, I.D. and Weetman, A.P. (1997) Identification of Ovarian Antibodies by Immunofluorescence, Enzyme-Linked Immunosorbent Assay or Immunoblotting in Premature Ovarian Failure. Human Reproduction, 12, 2617-2622. http://dx.doi.org/10.1093/humrep/12.12.2617

[9] De Vos, M., Devroey, P. and Fauser, B.C. (2010) Primary Ovarian Insufficiency. The Lancet, 376, 911-921. http://dx.doi.org/10.1016/S0140-6736(10)60355-8

[10] Nelson, L.M. (2009) Clinical Practice. Primary Ovarian Insufficiency. The New England Journal of Medicine, 360, 606-614. http://dx.doi.org/10.1056/NEJMcp0808697

[11] Practice Committee of the American Society for Reproductive M (2015) Testing and Interpreting Measures of Ovarian Reserve: A Committee Opinion. Fertility and Sterility, 103, e9-e17. http://dx.doi.org/10.1016/j.fertnstert.2014.12.093

[12] Ulug, P., Oner, G., Kasap, B., Akbas, E.M. and Ozcicek, F. (2014) Evaluation of Ovarian Reserve Tests in Women with Systemic Lupus Erythematosus. American Journal of Reproductive Immunology, 72, 85-88. http://dx.doi.org/10.1111/aji.12249

[13] Brougham, M.F., Crofton, P.M., Johnson, E.J., Evans, N., Anderson, R.A. and Wallace, W.H. (2012) Anti-Mullerian Hormone Is a Marker of Gonadotoxicity in Pre- and Post-Pubertal Girls Treated for Cancer: A Prospective Study. The Journal of Clinical Endocrinology and Metabolism, 97, 2059-2067. http://dx.doi.org/10.1210/jc.2011-3180

[14] Anderson, R.A. and Cameron, D.A. (2011) Pretreatment Serum Anti-Mullerian Hormone Predicts Long-Term Ovarian Function and Bone Mass after Chemotherapy for Early Breast Cancer. The Journal of Clinical Endocrinology and Metabolism, 96, 1336-1343. http://dx.doi.org/10.1210/jc.2010-2582

[15] Malheiro, O.B., Rezende, C.P., Rocha, A.L., Del Puerto, H.L., Ferreira, G.A. and Reis, F.M. (2014) Regular Menstrual Cycles Do Not Rule out Ovarian Damage in Adult Women with Systemic Lupus Erythematosus. Gynecological Endocrinology: The Official Journal of the International Society of Gynecological Endocrinology, 30, 701-704. http://dx.doi.org/10.3109/09513590.2014.922949

[16] Lawrenz, B., Henes, J., Henes, M., Neunhoeffer, E., Schmalzing, M., Fehm, T. and Kïtter, I. (2011) Impact of Systemic Lupus Erythematosus on Ovarian Reserve in Premenopausal Women: Evaluation by Using Anti-Muellerian Hormone. Lupus, 20, 1193-1197. 